\title{
Correction to: Worshipping the Tiger: Modeling Non-use Existence Values of Wildlife Spiritual Services
}

\author{
Adrian A. Lopes ${ }^{1} \cdot$ Shady S. Atallah ${ }^{2}$ \\ Published online: 29 January 2021 \\ (c) Springer Nature B.V. 2021
}

\section{Correction to: Environmental and Resource Economics (2020) 76:69-90 https://doi.org/10.1007/10640-020-00416-1}

The article was published with several textual and typographical errors introduced during proofing.

Under Section 1, Introduction, the following textual errors should be identified and noted:

1. The text provide, should be noted as provides.

Under Section 2, The Existence Value Model, the following textual errors should be identified and noted:

2. The text tsource, should be noted as to source.

3. The text vue, should be noted as value.

4. The text foowing, should be noted as following.

5. In the same section the following should be noted:

Equation (8) implies that in steady-state, the resource is neither growing nor diminishing over time or that harvest equals the growth in each $t$. Using the fact that $\rho=1 /$ $(1+\delta)$ and rearranging Eqs. (6), (7), and (8) we derive Eq. (9).

6. The text, rource, should be noted as resource.

7. The text $t$, should be noted as than.

8. The capitalized text, Wean, should be noted as We can.

The original article can be found online at https://doi.org/10.1007/10640-020-00416-1.

Shady S. Atallah

satallah@illinois.edu

Adrian A. Lopes

alopes@aus.edu

1 Department of Economics, American University of Sharjah, University City, PO Box 26666, Sharjah, UAE

2 Department of Agricultural and Consumer Economics, University of Illinois Urbana-Champaign, 1301 W. Gregory Dr., Urbana, IL 61801, USA 
9. In the same sentence, the text, mel should be noted as, model.

10. In the same section, the following sentence should be noted as follows:

In Section 3.1, we will assume functional forms for $\pi(\bullet), \mathrm{V}(\bullet)$, and $\mathrm{F}(\bullet)$, and calibrate the model for the tiger population in the BRT tiger reserve in southwestern India with the resident Soligas tribe.

Under Section 3 Applying the Model to a Tiger Population and a Resident Tribe.

11. The first two opening sentences should read as follows:

In this section, we will apply the model to the Soligas tribe and the tiger population in the BRT. Our baseline scenario is taken to represent the current situation in the BRT wherein the Soligas have community rights in accordance with the Forest Rights Act and all poaching is subject to a penalty as per the Indian Wildlife Protection Act.

12. The second paragraph in the same section should read as follows:

We define $p$ as the per unit price of the harvested resource and $c$ as a harvest cost parameter. Harvesting the tiger resource attracts a poaching penalty, and with this penalty in place, there is a chance that the harvester would be caught poaching by conservation authorities. We presume that a greater amount of illegal activity increases the chance of being caught (Copeland and Taylor 2009). We consider an exponential probability density function (Pishro-Nik 2014) of the following form: $\omega\left(Y_{t}, X_{t}\right)=\left(Y_{t} / X_{t}\right) e^{\left(\theta\left(1-Y_{t} / X_{t}\right)\right)}$. In this probability density function we have $\theta \leq 0$ and $X_{t} \geq Y_{t} \geq 0$, and it indicates that as illegal harvest increases so does the chance of being caught by conservation authorities. If the harvester is caught then a penalty of $\$ B$ is imposed on her, and this enters as an expected payment that is deducted from her private net benefit.

13. The text Fxistence should be noted as For the existence.

14. The fourth paragraph should read as follows:

For the discrete-time infinite horizon optimization problem in (10), one can set up the Lagrangean $\left(L^{B}\right)$, derive the first-order necessary conditions in steady-state, and substitute $Y=F(X)=r X(1-X / K)$ to get the fundamental equation of renewable resources, $(\phi(X, F(X) ; \delta, B) \equiv 0)$ in Eq. (11); this fundamental equation accounts for the existence value within the tribe manager's decision framework.

15. After Eq. (11.1), the first sentence should read as follows:

In Eq. (12), we consider the modalities of an alternative policy scenario where the tribe possesses land rights but there is no poaching penalty imposition. We therefore assume that $\pi\left(X_{t}, Y_{t}\right)=\left(p Y_{t}-(c / 2) Y_{t}^{2} / X_{t}\right)$.

16. After Eq. (12), the second sentence should read as follows:

The tribe manager explicitly accounts for the existence value in (13), whereas (13.1) represents the fundamental equation of renewable resources without the addition of existence value and therefore solves for the outcome of a harvester.

17. In Table 1, the text Tence should be noted as Existence.

18. After Table 1, the last sentence of the first full paragraph should read:

The BRT Tiger Reserve is $540 \mathrm{~km}^{2}$ (KFD 2017), which implies a carrying capacity of $K=\frac{540}{6.25}=86$ tigers. The BRT Tiger Reserve had approximately 35 tigers around 2010 (Varma 2015); accordingly, we assume $X_{0}=35$.

19. After Table 1, the text Asiderable should read A considerable amount.

20. The text Fstly should read Firstly.

21. The last paragraph of this section's introduction should read: 
The numerical exercise will reveal if the system is already in a steady-state or not by seeing if the observed $X_{0}=X^{*}$. Secondly, we will use the iterative map $\left[X_{t+1}=X_{t}+F\left(X_{t}\right)-Y_{t}\right]$ to simulate the evolution of $X(\mathrm{t})$ and examine if its approach path converges to $X^{*}$ if the initial stock is not yet at the steady-state, i.e. if $X_{0} \neq X^{*}$. We will examine the approach paths that represent tiger population dynamics under the various policy scenarios.

\section{Section 3.1}

22. The title of this section should read: Tiger Dynamics Under Different Policy Scenarios.

23. The first paragraph of Sect. 3.1.1 should read:

The first policy scenario that we consider is one where the resident Soligas tribe has secure property rights to the tiger resource, faces penalty imposition for poaching, the tribe collectively manages the resource in a way that is not myopic, and where the existence value is included in the optimization problem. This scenario is designed to represent the current situation in the BRT. Equation (11) can be numerically solved to derive steady-state values $\left(X^{*}, Y^{*}\right)$ for the parameters in Table 1. $B=\$ 769$ and $\theta=-5$ are the penalty function parameters where $B$ is the penalty amount and $\theta<0$ yields a positive probability, i.e. $e^{\left(\theta\left(1-Y_{t} / X_{t}\right)\right)} \geq 0$. We accordingly derive $X^{*}=56.557$ and $Y^{*}=F\left(X^{*}\right)=0.968$. We use Solver at the same time to back out an existence value weight of $\beta=167.50$, which yields a steady-state existence value of $\beta \ln \left(X^{*}\right)=675.92$ ${ }^{1}$ This numerical estimate of $\beta$, which is backed out using a calibration that represents the current situation in the BRT, is essentially a revealed preference parameter that can be used to estimate the tiger's spiritual ecosystem service value from the perspective of the Soligas tribe manager. Using this perspective, the $\beta$ value is unique to the Soligas, given the current situation in the BRT. Thus, $\beta=167.50$ would be applicable to any policy scenario, and as such would be considered a given value that represents the Soligas preferences, regardless of policy regime shifts.

24. The text Aing should read After having.

25. Eq. (14) should read:

$$
\begin{aligned}
\underset{\left\{Y_{t}\right\}_{0}^{T-1}}{\operatorname{maximize}} \mathbb{E}\left[W^{B}\right]= & \sum_{t=0}^{T-1} \rho^{t}\left[p Y_{t}-(c / 2) Y_{t}^{2} / X_{t}-\left(Y_{t} / X_{t}\right) e^{\left(\theta\left(1-Y_{t} / X_{t}\right)\right)} \cdot B+\beta \ln \left(X_{t}\right)\right] \\
& +\rho^{T-1}\left\{p r X_{T}\left(1-X_{T} / K\right)-\frac{c}{2 X_{T}}\left[r X_{T}\left(1-X_{T} / K\right)\right]^{2}-B \cdot r\left(1-\frac{X_{T}}{K}\right) e^{\left(\theta\left(1-r\left(1-X_{T} / K\right)\right)\right)}+\beta \ln \left(X_{T}\right)\right\} / \delta
\end{aligned}
$$

subject to $X_{t+1}=X_{t}+r X_{t}\left(1-X_{t} / K\right)-Y_{t}$ and $X_{0}>0$ given.

26. The paragraph after Eq. (15) should read:

The final function can be thought of as the value of maintaining $X_{T}$ for $t=T, T+1, \ldots, \infty$ by harvesting $Y_{t}=r X_{T}\left(1-X_{T} / K\right)$ for the rest of time in steadystate. With $Y_{t}=r X_{T}\left(1-X_{T} / K\right)$ being a constant, it can be factored out of the infinite series with the present value converging to $\psi\left(X_{T}\right)$. Using the parameters in Table 1, assuming an initial value of $X_{0}=35, T=60$ years, and assigning initial value guesses for $\left\{Y_{t}\right\}_{t=0}^{t=59}=0.10$, we numerically estimate the approach paths of $X(t)$ and $Y(t)$ over the horizon of $T=60$ years. Using Solver, we find convergence to a maximum value for the objective function, $\mathbb{E}\left[W^{B}\right]=8691.1$. Moreover, we note that $X_{t}=56.5$ as

\footnotetext{
1 This exercise circumvents the arbitrariness of an assumed value of $\beta$ and the possibility of multiple steady states based on this assumption.
} 
$t \rightarrow 60$, which implies that the approach path of the resource stock indeed approaches the steady-state value of $X^{*}$ as derived from Eq. (11). This approach path of $X_{t}$ is shown in Fig. 2. This policy result would correspond to the implicit equation $\phi_{2}(X, Y ; \delta)$ or $\phi_{3}(X, Y ; \delta)$ in Fig. 1 that yielded steady-state equilibriums over time. The dotted line in Fig. 2 depicts the approach path of $X_{t}$ without the existence value; in this scenario the existence value, $\beta \ln \left(X_{t}\right)$, drops out of the optimization framework according to Eq. (11.1). The dotted line approach path allows one to examine the effect of excluding existence value from the tribe manger's decision framework. (Note: Following this paragraph, the incomplete sentence "proach paths of $X(t)$. with and without existence value in the presence of secure property rights for Soligas and poaching penalties." should be ignored as it is a repeat of the title of Fig. 2.)

27. The paragraph after Eq. (17) should read:

This first-order necessary condition can be solved numerically using Solver to equate it to zero by finding the harvest $Y_{t}$, with $X_{t}$ treated as a parameter. One would note that the existence value $\beta \ln \left(X_{t}\right)$ does not feature in this myopic harvester's decision as per Eq. (17). Once again, the initial resource stock is $X_{0}=35$ tigers. Once Solver yields the initial harvest, $Y_{0}$, the iterative map $\left[X_{t+1}=X_{t}+F\left(X_{t}\right)-Y_{t}\right]$ is called upon to derive $X_{1}$, and the exercise is repeated for $t=1,2, \ldots, T$. In the approach path shown in Fig. 3, we observe that the resource stock $X_{t} \rightarrow 0$ and tiger extinction in the BRT occurs in 49 years. Extinction occurs in consonance with the implicit equation, $\phi_{1}(X, Y ; \delta)=0$, that yielded localized tiger extinction in the phase diagram of Fig. 1. This implies that extinction is optimal when poaching is driven up by the harvester's myopic behavior with a high discount rate $\delta$ that results in $X$ growing slower than the offtake.

Section 3.1.3

28. The title of this section should read:

Secured Property Rights with no Poaching Penalty.

29. The first paragraph of this section should read:

The third policy scenario that we consider is one where the resident Soligas tribe has secure property rights to the tiger resource and the tribe collectively manages the resource in a way that is not myopic as under Scenario 3.1.1, and that includes the existence value in the optimization problem. The departure from Scenario 3.1.1 is the absence of a poaching penalty. The parameter values in Table 1 are substituted into Eq. (13) to numerically solve for the steady-state values of resource stock $\left(X^{*}\right)$ and harvest $\left(Y^{*}\right)$ by using Solver. Under Scenario 3.1.1, we backed out the value of $\beta$ as 167.50; this weight is the preference parameter of the Soligas tribe as to the tiger's existence. Using Solver, the steady-state equilibrium values we derive are $X^{*}=56.003$ and $Y^{*}=F\left(X^{*}\right)=0.976$, with $\beta$ set at 167.50. This situation would correspond to the implicit equation $\phi_{2}(X, Y ; \delta)$ or $\phi_{3}(X, Y ; \delta)$ in Fig. 1 that yielded steady-state equilibriums over time. The existence weight of $\beta=167.50$ yields a steady-state existence value of $\beta \ln \left(X^{*}\right)=674.27$; this value is not meaningfully different from the 675.92 derived under Scenario 3.1.1. Next, we examine the resource's approach path when $X_{0} \neq X^{*}$ by using the final function method described earlier. In order to do this, we revisit the tribe manager's optimization problem listed in Eq. (12) and rewrite it as Eq. (18).

30. The sentence before Eq. (19) should read: 
The objective function $W^{f}$ is the sum of the present value of net benefits over time $t=0,1,2, \ldots, T-1$ and a final function, Eq. (19); i.e., the value of maintaining $X_{T}$ for $t=T, T+1, \ldots, \infty$ by harvesting $Y_{t}=r X_{T}\left(1-X_{T} / K\right)$ for the rest of time in steadystate.

31. The paragraph after Eq. (20) should read:

The first-order necessary condition yields the optimal harvest as $Y_{t}=(p / c) X_{t}$ in each $t$. Note the similarity with scenario 3.1.2 under which the existence value $\beta \ln \left(X_{t}\right)$ does not feature in the myopic harvester's decision. We use the parameters from Table 1 to derive the approach path of $X(t)$ in this policy scenario, i.e., without secure property rights to the sacred tiger resource. This approach path is shown in Fig. 5 with a starting value of $X_{0}=35$ tigers. We observe that the resource stock $X_{t} \rightarrow 0$ and localized tiger extinction in the BRT occurs as $t \rightarrow 38$ years. The absence of a poaching penalty in this policy scenario hastens extinction by 11 years compared to what was observed in the approach path in Fig. 3. Likewise, extinction occurs in consonance with the implicit equation, $\phi_{1}(X, Y ; \delta)=0$, in Fig. 1 .

32. In Table 2, the text oaching should read poaching. (Note: Please refer to the PDF document supplied for instances where periods that were added during the production process - after variable names and equations in the middle of sentences - should be removed throughout the article.)

Publisher's Note Springer Nature remains neutral with regard to jurisdictional claims in published maps and institutional affiliations. 